\title{
Performance Analysis of Inter and Intra IMS Network
}

\author{
A Bagubali, ${ }^{1}$ Ankit Agarwal, ${ }^{2}$ V Prithiviraj ${ }^{3}$ and P. S Mallick ${ }^{4}$ \\ 1,2,4 Vellore Institute of Technology University, Vellore-632014, Tamilnadu, India \\ bagubali@vit.ac.in; ankit.agrwl80@gmail.com; psmallick@vit.ac.in \\ ${ }^{3}$ Rajalakshmi Institute of Technology, Chennai, Tamilnadu, India \\ profvpraj@gmail.com
}

Received 19 August 2014; Accepted 19 November 2014; Publication 19 March 2015

\begin{abstract}
The IP Multimedia Subsystem (IMS) is a network architecture that consists of different network elements linked by standard interfaces. It facilitates the delivery of multimedia services based on Internet Protocol(IP) and makes the integration of services with the internet much easier. It is a method to achieve the convergence of fixed and mobile communication devices. Optimized transmission of voice, data and video communications among the users can be achieved using IMS architecture, independent of the user's location and devices. IMS enables the user to use different services in a more efficient way in terms of energy saving. Using this technology, it is possible to make the network distribution in a better way. It reduces the energy consumption because of the capability to switch network whenever possible. This paper focuses on establishment of end to end session between two IMS terminals and analysing the performance for different applications. The performance is measured on the basis of traffic parameters and call setup delay. We have compared Inter and Intra IMS network based on a model that ensures the delivery of services without any data loss and with minimum delay.
\end{abstract}

Keywords: IMS, SIP, HSS, VoIP, QoS.

Journal of Green Engineering, Vol. 4, 175-194.

doi: 10.13052/jge1904-4720.431

(c) 2015 River Publishers. All rights reserved. 


\section{Introduction}

The world of communication is emerging towards the trend that supports integration of user with the outside world in a much easier way. IMS solves the continuing demand of interoperability where the user can enjoy wide range of services despite their location and access medium. Interoperability is achieved by the convergence of fixed and mobile communication devices and networks. Fixed-mobile convergence means a device can change its connection from one network to another network, say wired to wireless network. While using IMS at both the terminals, it is easier for the users to adopt any network without any interrupt in the services. The application servers used in IMS, performs the management operations, that basically takes care of energy consumption. It has become the biggest challenge in today's generation to achieve less energy consumptive method while using multimedia. IMS mainly uses Session Initiation Protocol (SIP), as the protocol for unifying the applications. It is responsible for VoIP call setup and handling [1]. A standard IMS architecture comprises of many SIP servers and Home Subscriber Server(HSS) also known as user database [2]. HSS contains information about the user subscription and provides subscriber's location and IP information. The purpose of SIP is to initiate the session among user agents. The proxy servers used in IMS are the major part its architecture. They are collectively given the name as Call Session Control Functions (CSCF). These functions can be further classified as ProxyCSCF, Serving-CSCF, and Interrogating-CSCF [3]. $\mathrm{P}-\mathrm{CSCF}$ is a starting point of contact with which the user initiates to send the call request. It provides authentication to an user and inspects the signal ensuring that the IMS terminal do not misbehave. S-CSCF is a SIP server responsible for registrations by verifying the address assigned to an user. It takes a decision upon choosing the application server to which the SIP message will be forwarded. I-CSCF is responsible for contacting HSS to take the user location and forwarding the corresponding response to S-CSCF.

The rest of this paper has been designed as follows: Next subsections describe about the architecture and different processes involved related to IMS. Section 2 discusses the related work that has been done in past. The model that has been used in this paper, is described in Section 3. Simulation results have been presented in Section 4 and finally the conclusions are made in Section 5.

\subsection{IMS Architecture}

The IMS architecture includes three separated layers, each functioning independently. The three layers are named as: 
- Transport and End Point Layer: It is responsible for changing the format of incoming media from analog $\backslash$ digital to real time transfer protocol and SIP protocol. This layer separates the access layers from the IP network above it. It is responsible for assigning the IP address and registering of devices with the upper layers.

- Control and Session Layer: This layer forms the logical connections between various network elements. As suggested by the name itself, it controls the authentication, routing and traffic between transport and service layer. It also provides an interface between service layer and other services. Layer consists of two important IMS network elements i.e. CSCF and HSS. CSCF manages the interaction among the users with the help of SIP servers. HSS contains the whole information about the user subscription which helps in authenticating the user with the network.

- Application Services Layer: The application services layer consists of several Application Servers, responsible for performing different functions on user's session. This layer is responsible for deployment of new services in the IMS network with the existing services.
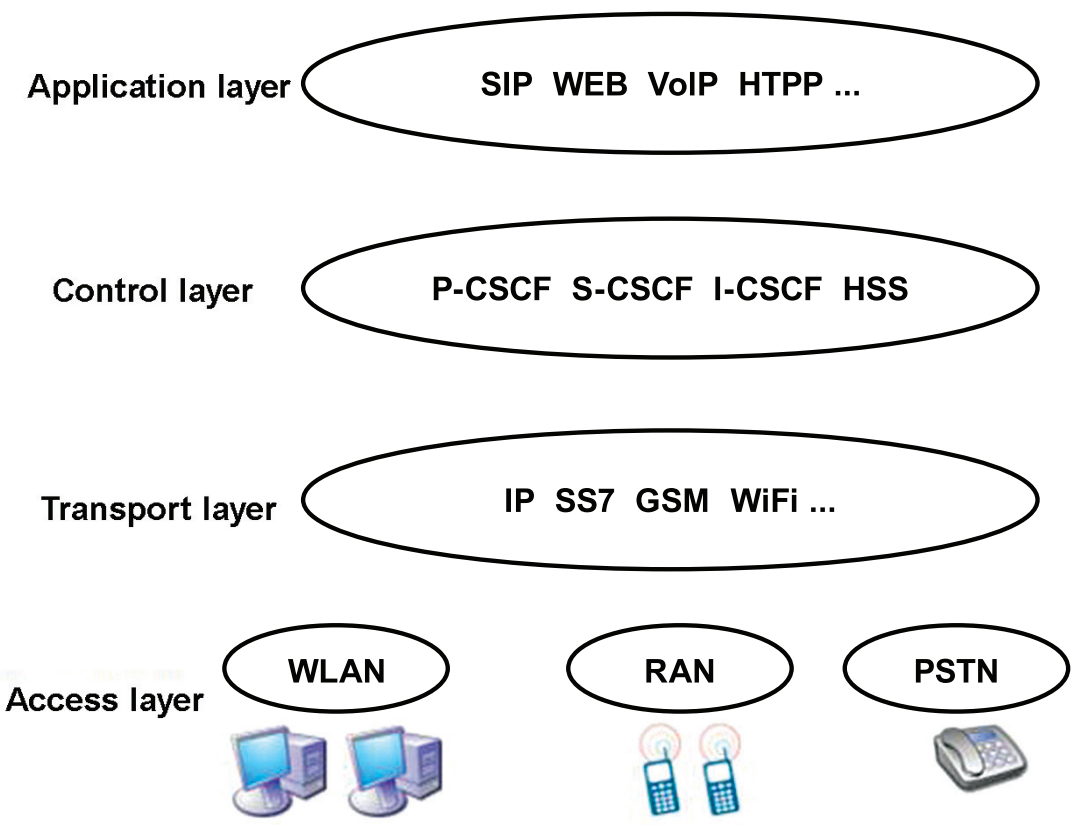

Figure 1 IMS three layered architecture 


\subsection{Need of IMS}

As stated earlier, IMS provides an integrated architecture for the transmission of different multimedia services over internet protocol. The main motive behind using the IMS architecture is to manage the network in a much easier way. IMS provides the opportunity to network operators for delivering more reliable and profitable multimedia services with the existing services. It provides the facility of migration from circuit switching services to packet switching domain. Different users can be connected as IMS terminal and the initiated call can be easily handed off from one network to another network without any interruption. IMS architecture is access independent therefore the services are delivered independent of access technology [4]. It supports for roaming between different networks. IMS has provided the options to combine ongoing voice call with different multimedia elements like sharing photos and videos while talking. IMS enables the single integrated network for all the access types. Therefore the cost has come down for the service providers. The IMS compatible systems are designed to support multiple application servers which means that same architecture can be reused for new services that is made to focus on actual service to be provided. The authentication process is more simpler and has been standardized in IMS as compared to non-IMS architecture. Once the subscriber is authenticated through IMS, it can access all the IMS services. In non-IMS scenario each service has its own way to authenticate the user. Conclusively, IMS provides a better secure connection with the outside world giving the facility to change the communication mode smoothly.

\subsection{IMS Challenges}

IMS is a new emerging technology towards the evolution of user integration with the internet. There are several challenges and objectives that can be addressed over deployment of IMS architecture. IMS has emerged as a solution to manage the network smoothly. Some of the challenges are mentioned below related to the IMS architecture

- Quality Of Service (QOS): One of the major issues in implementing IMS architecture is to enhance the Quality of service parameters, so that user can meet its demands regarding bandwidth and delay requirements. It is essential to provide secure connection to the user that protects it from creating a traffic load for other users.

- Multiple Services: As stated earlier, one of the important feature of IMS is to enable different services simultaneously. IMS is mainly designed to achieve this purpose so that user can be able to enjoy wide range of 
services at a time. Handling of multiple services without any delay and interrupt during the call flow has come up as a challenge before IMS. Another issue is regarding the security of these services, IMS should be able to provide a secure connection for each of the services.

- Authentication: IMS architecture uses a completely different methodology to provide authentication to a set of users. Authentication process needs to be strong in terms of security so that private data cannot be reached to malicious agents or unethical hackers. The registration request to IMS terminal has to be properly examined by the protocols used so that unidentified user can be easily ignored.

- Energy Saving: The major issue in today's world is related to energy. Main challenge is to develop a technology considering the environmental requirements. During the wireless transmission of any media, a lot of energy is consumed in terms of battery power. IMS provides the best network switching mechanism which enables it to reduce energy consumption whenever required.

\subsection{IMS Registration and Session Establishment}

In order to initiate a call among the users, IMS architecture must know where the user(User Equipment(UE)) is located. All the users must register to the network during the activation. If the user changes its location it must reregister to the network so that updated location can be retrieved. During the registration process, the request is sent to $\mathrm{P}-\mathrm{CSCF}$, which is the initial point to make contact with another user. On receiving the request, this SIP proxy needs to locate I-CSCF to know about the user's location and other information. I-CSCF sends a query to HSS in order to get the subscription information about the user. Once the registration request reaches to S-CSCF, it challenges the user by sending an Unauthorised response, so that it can receive the authorisation data from the user. This data is matched with the data available with HSS, and if verified, the positive authentication response is reached back to the user.

Once the registration process gets completed, if anyone wants to establish a session with user at the other end, it sends a SIP invite message to the first point of contact i.e. P-CSCF. After receiving invite message, it checks for the location of called user, whether it belongs to the same network or not. If network is found to be same for both calling and called party, then P-CSCF directly contacts to the assigned S-CSCF for called user. Invite message is sent to the S-CSCF, and now S-CSCF will be responsible for the arrival of invite message to the final receiver. But, if the called party belongs to other IMS 


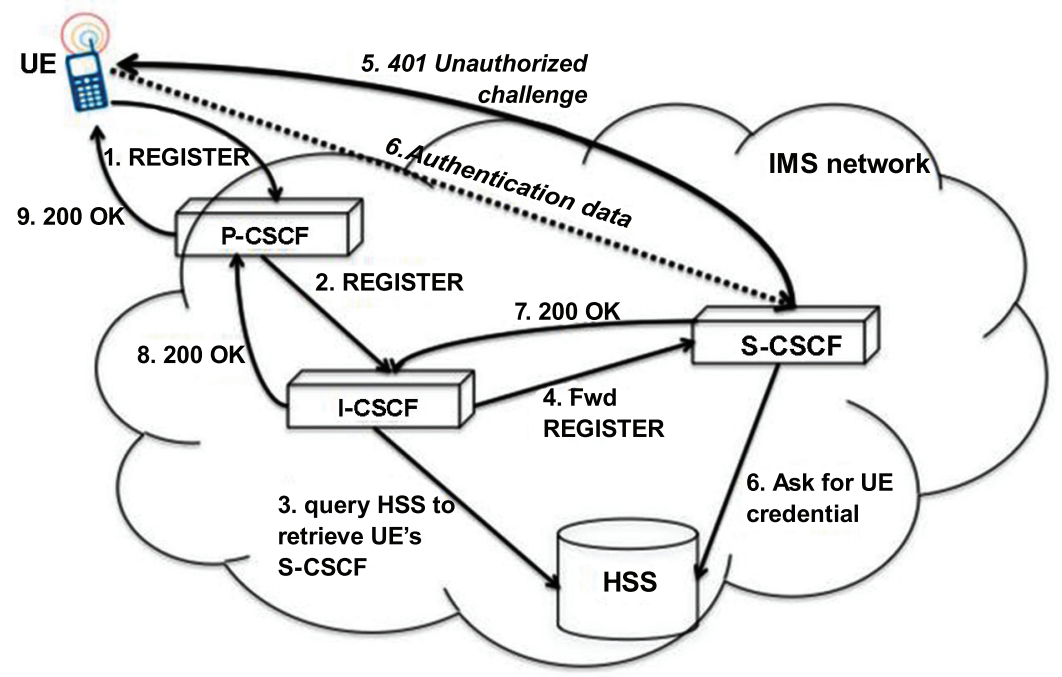

Figure 2 IMS registration

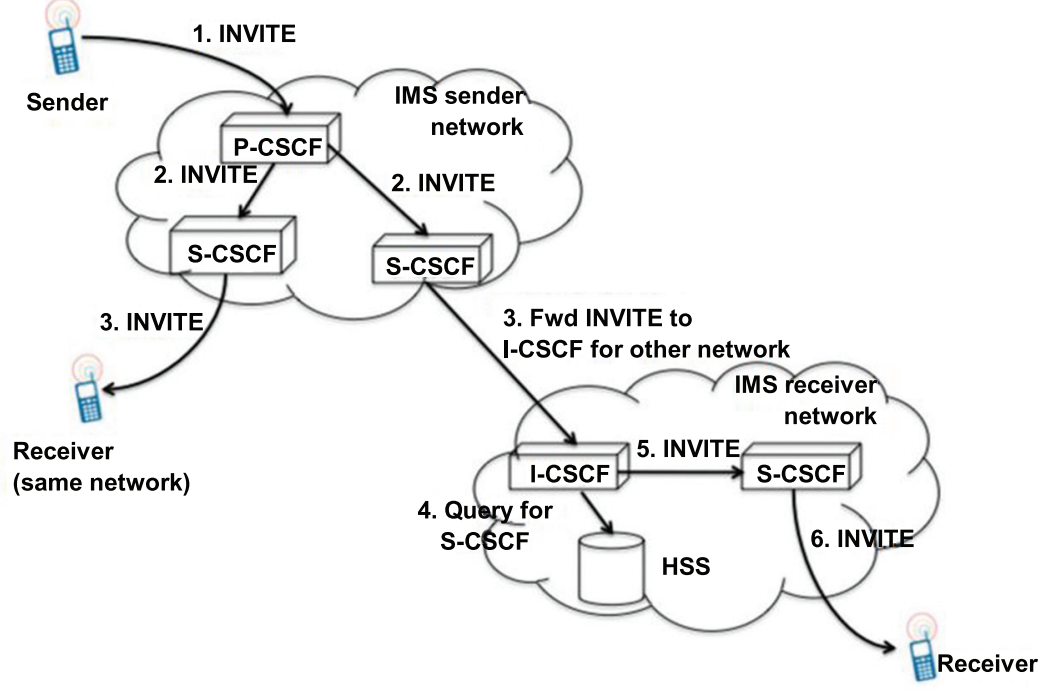

Figure 3 IMS session establishment

network, then P-CSCF has to locate corresponding I-CSCF of the external network, so that location of called party can be retrieved. I-CSCF contacts to HSS that may contain the user's information. If the user has not registered to 
the network or belong to some other network then HSS returns NOT FOUND response to the query. In case when the user's location is found in HSS, the Invite message is sent to S-CSCF and finally delivered to the receiver. The receiver then sends an acknowledgement to caller party, and at this point SIP session is successfully established [5].

\section{Related Work}

Most of the previous work related to IMS has focussed mainly on protocol development and complexity of SIP. However, to set up an uninterrupted connection it is important to take into account, the call setup delay. This analysis of delay parameter will be able to predict the data loss during the hand off process from one network to another network. Research of Eyers et al [6] was based on SIP call setup delay. It mainly focuses on data loss due to UDP. Kist et al [7] presented the signalling delays, assuming the queuing delay to be less than 5ms. V.S. Abhayawardhana, R. Babbage [8] presented a traffic model for the IP multimedia subsystem. In this model, signalling traffic was created to and from HSS in three processes i.e. registration, session setup and subscription. In [9], the authors have analyzed the SIP IMS network based on signalling delays, and their analysis was based on real time server traffic. Other researches has mainly concentrated on network performance evaluation based on different network parameters [10-12]. M. Melnyk and A. Jukan [13], studied the call setup efficiency in IP wireless networks. It presented the session forming process in IMS, where both the source and destination node are using same technology standards.

\section{Description of the Model}

To evaluate the performance of IMS network model and to solve the challenges related to it, we have designed and tested the network model in OPNET modeler 14.0. The network is designed in such a way that all the intermediate proxy servers have been used to complete the process of session establishment among the users. Two IMS networks are established in different regions, and three users are made to register with each IMS terminal. The designed network basically consists of SIP proxy servers at each end, connected with the Ethernet routers. As mentioned earlier, intermediate proxy servers are P-CSCF, S-CSCF, I-CSCF, each playing different roles in establishing the session among two users. Since, it's a internetwork scenario, we have used IP cloud to make a connection between the two networks. Traffic has been created by 


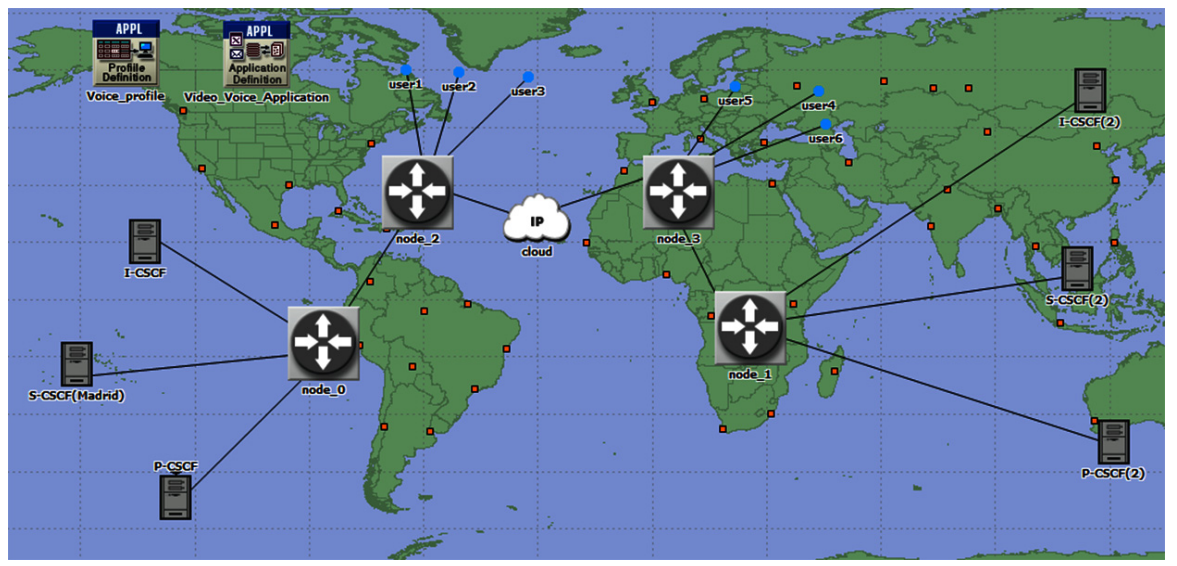

Figure 4 IMS internetwork scenario

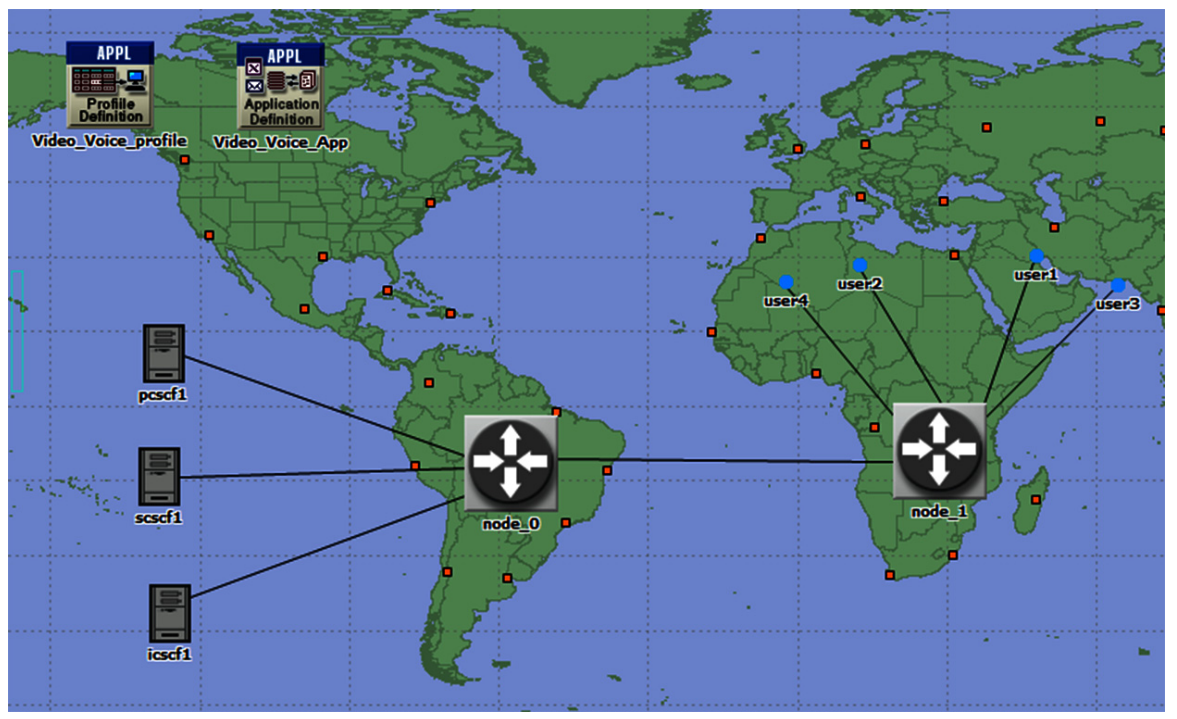

Figure 5 IMS intranetwork scenario

making use of different applications with VoIP. In this model, we have used Video Conferencing as secondary application which basically creates traffic while transmitting voice packets from one end to another.

If any user wishes to make a contact with the user located in same network, then the intermediate server I-CSCF will directly send invite request to the corresponding S-CSCF. There will be no need to send a query to HSS for 
Table 1 Applications

\begin{tabular}{lll}
\hline Applications & \multicolumn{1}{c}{ Attributes } \\
\hline Voice Application & Encoder Scheme & PCM quality speech \\
& Voice Frames/packet & 5 \\
& Compression Delay & $0.02 \mathrm{sec}$ \\
Video Conferencing & Type & Low resolution video \\
& Frame Interarrival & 10 frames/sec \\
& Frame size & $128 \times 120$ pixels \\
\hline
\end{tabular}

the user location as the called user belongs to same network. To test the performance of session establishment within the same network, we have created another scenario containing two users registered to the same IMS network. All the attributes and applications are kept same as previous scenario, the only change that has been made is, only one IMS network is present instead of two. Since, it's a intranetwork scenario, there is no need to use IP cloud for the connection setup. Only Ethernet routers have been used to connect the proxy servers and the users.

\subsection{Application Services}

In order to generate the traffic among the nodes, two simultaneous applications have been used in this model. One is voice application and the another application is video conferencing. Starting time of execution for both the applications have been given same, so that motive of designing IMS network can be successfully achieved.

\subsection{Mathematical Delay Model}

This section analyses the delay occurred at different stages for the designed IMS networks. IMS delay collectively comprises of three types of delay named as, processing delay, transmission delay and queuing delay.

$$
T=t_{p}+t_{t}+t_{q}
$$

where $T, t_{p}, t_{t}$ and $t_{q}$ represents total average IMS delay, processing delay, transmission delay and queing delay.

- Transmission delay: It is the delay occurred while transmitting signal message from UE to P-CSCF terminal From [13], it is assumed that there are total 8 messages included in the registration process from UE to P-CSCF. The IMS registration transmission delay can be written as: 


$$
t_{t}(i m s)=8 \times t_{t c p}
$$

- Processing delay: It is the delay due to packet wrapping and unwrapping in the network layer. The IMS processing delay can be written as:

$$
\begin{aligned}
t_{p}(i m s) & =4 \times t_{p-U E}+10 \times t_{p-P C S C F}+6 \times t_{p-I C S C F} \\
& +4 \times t_{p-H S S}+8 \times t_{p-S C S C F}
\end{aligned}
$$

Here, $t_{\mathrm{p}-\mathrm{UE}}, \mathrm{t}_{\mathrm{p}-\mathrm{PCSCF}}, \mathrm{t}_{\mathrm{p}-\mathrm{ICSCF}}, \mathrm{t}_{\mathrm{p}-\mathrm{HSS}}, \mathrm{t}_{\mathrm{p}-\mathrm{SCSCF}}$ represents the delay incurred for message processing at the respective nodes.

- Queuing delay: It is the delay occurred, because of queuing of packets at the network nodes. The queuing model is assumed to be of type M/M/1 queuing model, in which the arrivals occurs at rate $\lambda$ according to a poisson process and a single server serves the customers one at a time from the front. The IMS queuing delay can be written as:

$$
\begin{aligned}
t_{q}(i m s) & =4 \times E\left(t_{q-U E}\right)+10 \times E\left(t_{q-P C S C F}\right) \\
& +6 \times E\left(t_{q}-I C S C F\right)+4 \times E\left(t_{q}-H S S\right) \\
& +8 \times E\left(t_{q}-S C S C F\right)
\end{aligned}
$$

Here, $\mathrm{E}\left(\mathrm{t}_{\mathrm{q}-\mathrm{UE}}\right), \mathrm{E}\left(\mathrm{t}_{\mathrm{q}-\mathrm{PCSCF}}\right), \mathrm{E}\left(\mathrm{t}_{\mathrm{q}-\mathrm{ICSCF}}\right), \mathrm{E}\left(\mathrm{t}_{\mathrm{q}-\mathrm{HSS}}\right), \mathrm{E}\left(\mathrm{t}_{\mathrm{q}-\mathrm{SCSCF}}\right)$ represents the expected unit packet queing delay at the respective nodes.

As a combination of all the three delays, the total IMS registration delay can be written as:

$$
\text { Total IMS delay }=t_{t}(i m s)+t_{p}(i m s)+t_{q}(i m s)
$$

\section{Simulation Results}

\subsection{SIP Call Set UP Time}

Figure 6 analyzes the SIP call set up time for Inter/Intra IMS networks. It is observed that time taken in Intranetwork scenario is very less as compared to time taken in Internetwork scenario. This is due to the fact that in Intranetwork scenario both the users are located within the same network, that is why time taken to process the SIP call setup request is very less. While in Internetwork scenario users belong to different networks, therefore time taken is more, as the location of the called user has to be retrieved first and then the request is being processed. 


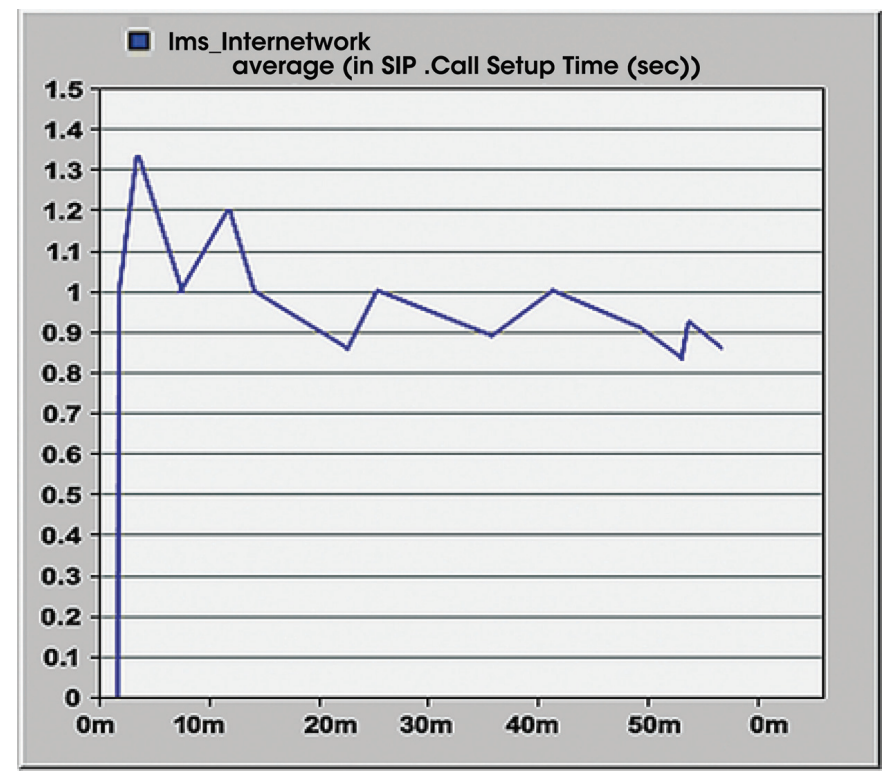

(a)

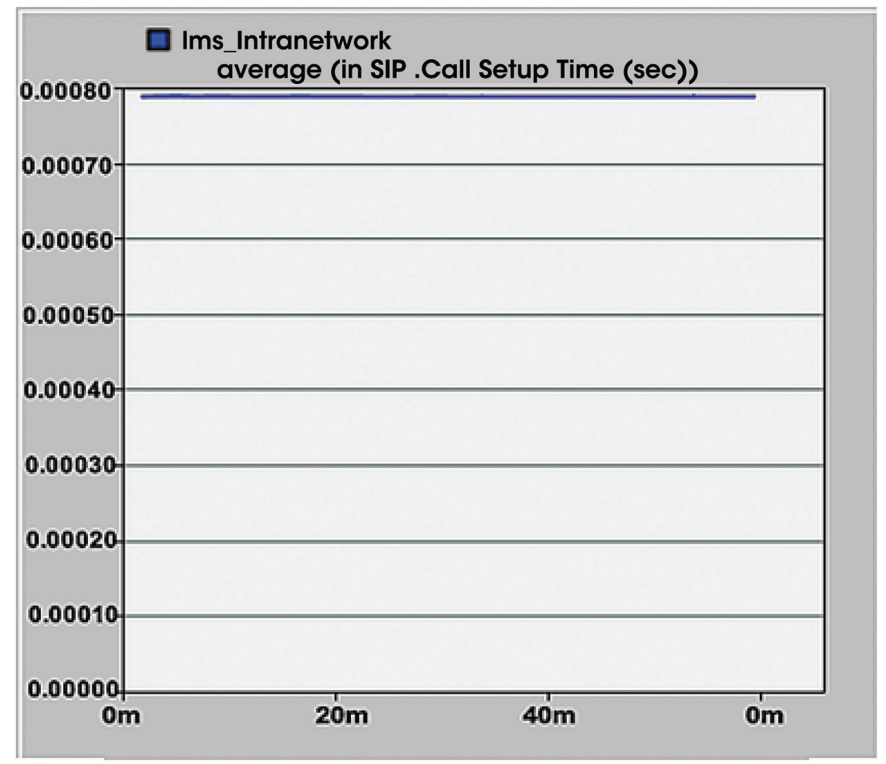

(b)

Figure 6 SIP call setup time (a) Internetwork (b) Intranetwork 


\subsection{Voice Traffic Sent and Traffic Received}

Figure 7 analyzes the voice traffic sent and traffic received in terms of packets/sec for the Intranetwork scenario and Internetwork scenario. It is observed that there is very less amount of packet loss during the transmission. So the efficiency for both the networks is achieved very high.

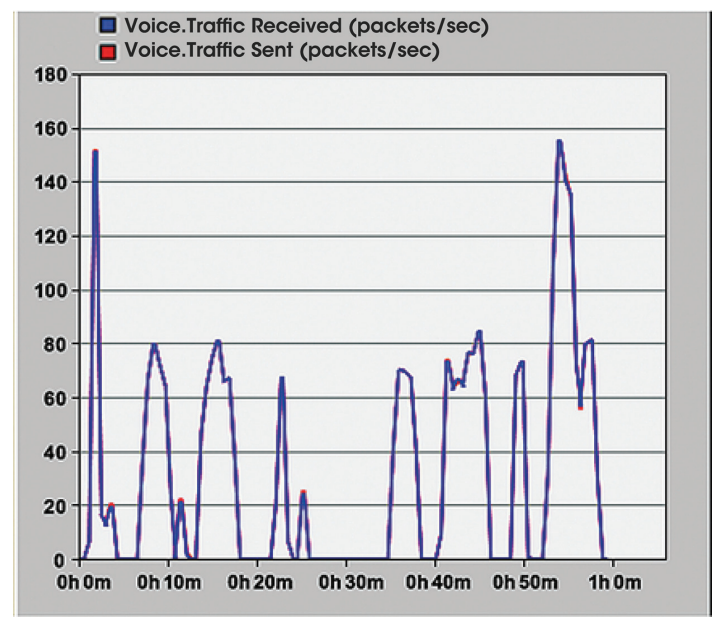

(a)

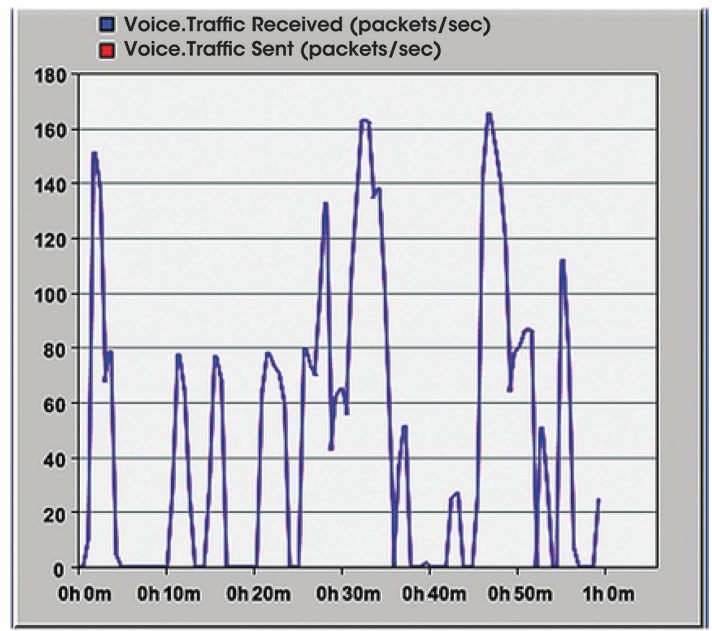

(b)

Figure 7 Voice Traffic Sent and Traffic Received (a) Internetwork (b) Intranetwork 


\subsection{Video Traffic Sent and Traffic Received}

Figure 8 analyzes the traffic sent and received for the video conferencing application in Intra/Inter IMS network. It is again observed that packet loss in both the networks is negligible. Therefore, it can be concluded that whenever the IMS network is used, simultaneous multimedia application can be transmitted/received without any data loss.

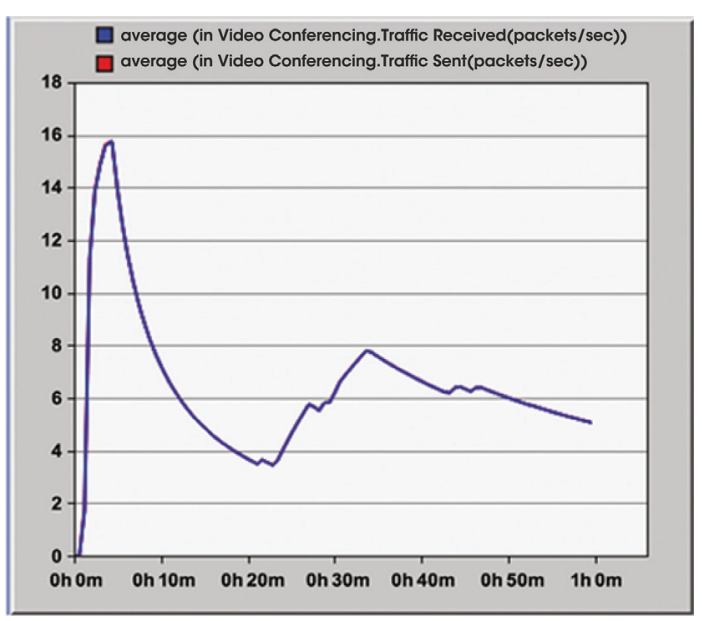

(a)

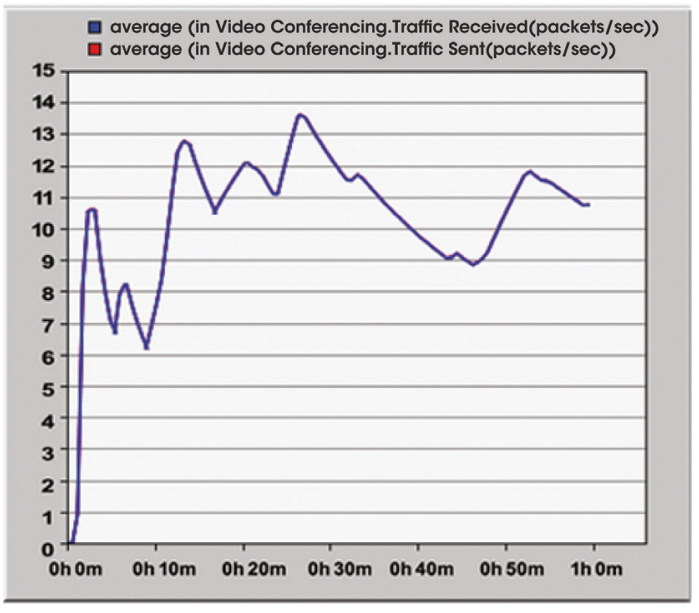

(b)

Figure 8 Video Traffic Sent and Traffic Received (a) Internetwork (b) Intranetwork 


\subsection{SIP UAC Active Calls and Call Duration}

Figure 9(a) compares the total number of UAC active calls for both the Inter/Intra network scenario. These are the calls which are activated form user1 to user2 after the SIP request is accepted at the terminal.

Figure 9(b) compares the active call duration for both the scenarios. Call duration is more in case of internetwork scenario as compared to intranetwork.

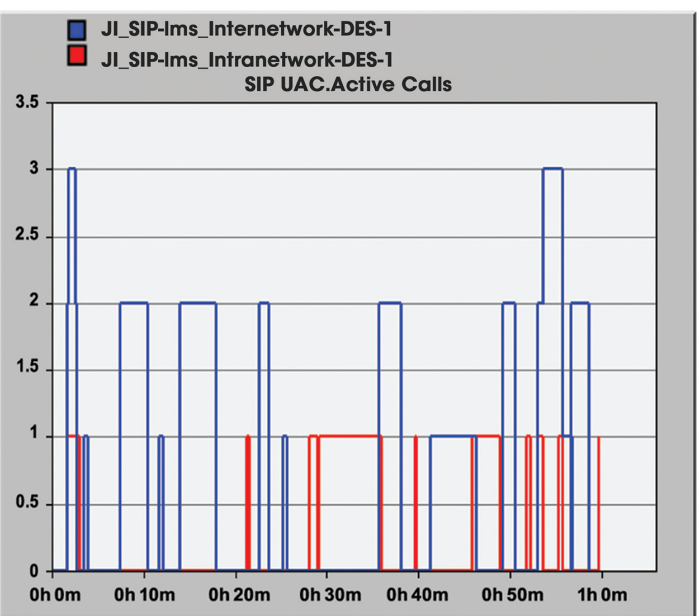

(a) SIP UAC Active Calls

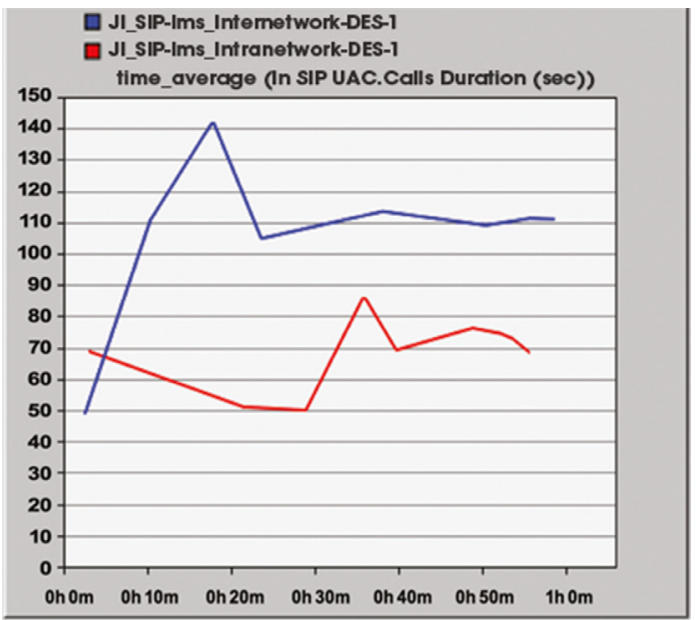

(b) ISP UAC Call Duration

Figure 9 SIP Call Information 


\section{Conclusion}

As already discussed in this paper, use of IMS network makes the hand off process much easier during the call transfer from one network to another. We have analyzed the performance of IMS network based on the call setup delay and traffic transferred from one user to another. In this paper IMS network has been designed in two phases .i.e. Intranetwork and Internetwork. We have simulated both the scenarios using OPNET modeler tool, that takes into account all the intermediate servers like S-CSCF, P-CSCF and I-CSCF. It has been concluded that time taken to setup a call is very less in intranetwork as compared to internetwork. Also we have seen that there is no data loss in both the networks for voice as well as video transfer. The use of IMS leads to a solution of energy consumption during wireless transmissions. It has become possible because protocols adopted by IMS mainly consider the node according to energy consumption and also handoff techniques are appropriately adopted by IMS to achieve the goal of energy saving wireless environment. The network switching mechanism in IMS has been designed in such a way that it always prefers to choose the network having high range with less energy consumption.

For future work, we are planning to integrate this IMS network model with other networks, to achieve the target of proper hand off while switching between different networks during a call. This IMS model may act as intermediate network, so that call can be transferred without any interruption.

\section{References}

[1] N. Rajagopal, M. Devetsikiotis, "Modeling and Optimization for the Design of IMS Networks", 39th Annual Simulation Symposium 2006, pp: 34-41.

[2] P. Agrawal, et. al, "IP Multimedia Subsystems in 3GPP I $\backslash \&$ 3GPP2:Overview and Scalability issues", IEEE Communications Magazine, January, 2008.

[3] Poikselka, M.; Mayer, G.; Khartabil, H.;Niemi, A. The IMS: IP Multimedia Concepts and Services in the Mobile Domain. England: WILEY, 448 p. ISBN-10 0-470-87113-X, 2004

[4] Camarillo, G.; García-Martín, M. A(2008). The 3G IP Multimedia Subsystem (IMS) Merging the Internet and the Cellular Worlds. Third Edition. England: WILEY, 618 p. ISBN-13978-0-470-51662-1

[5] 3GPP IP Multimedia Subsystem (IMS), 3GPP TS 23.228, 2013 
[6] T. Eyers and H. Schulzrinne. Predicting internet telephony call setup delay In IPTel 2000, First IP Telephony Workshop, Berlin, Germany, 2000.

[7] A. Kist and R. Harris. SIP signalling delay in 3GPP. In Proceedings of Sixth International Symposium on Communications Interworking of IFIP-Interworking 2002, Perth, Australia, October 13-16 2002.

[8] V.S. Abhayawardhana, R. Babbage, "A Traffic Model for the IP Multimedia Subsystem (IMS)", IEEE 65th Vehicular Technology Conference, 22-25 April 2007, pp. 783-787.

[9] J.-S. Wu and P.-Y. Wang. The performance analysis of SIPT signaling system in carrier class VoIP network. (AINA'03).

[10] S. Pandey, V. Jain, D. Das, V. Planat, R. Periannan, "Performance Study of IMS Signaling Plane", IP Multimedia Subsystem Architecture and Applications, International Conference, 6-8 Dec. 2007, pp.1-5.

[11] A. Munir, "Analysis of SIP-Based IMS Session Establishment Signaling for WiMax-3G Networks", Networking and Services, 4th International Conference, 16-21 March 2008, pp. 282-287.

[12] A. A Kist, RJ. Hark, "SIP signalling delay in 3GPP", IEEE 6th international Symposium on Communications Interworking of IFIP interworking, Fremantle WA, October 13-16, 2002.

[13] M. Melnyk and A. Jukan. On Signaling Efficiency for Call Setup in all-IP Wireless Networks. In Proceedings of IEEE International Conference on Communication (ICC'06), Istanbul, Turkey, June 2006.

\section{Biographies}

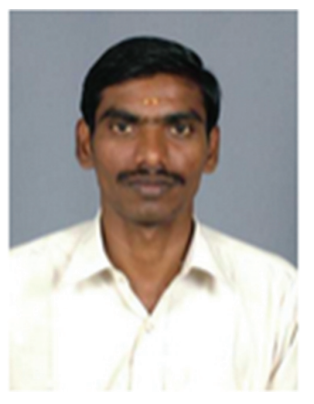

A. Bagubali obtained his B.Tech in electronics and communication engineering from VIT University, Vellore, in 2005, and M.Tech in communication engineering from VIT University, Vellore, in 2007. Currently he is senior 
assistant professor in VIT University, Vellore. His research interests include IP multimedia subsystem applied to $3 \mathrm{G}$ and $4 \mathrm{G}$ networks.

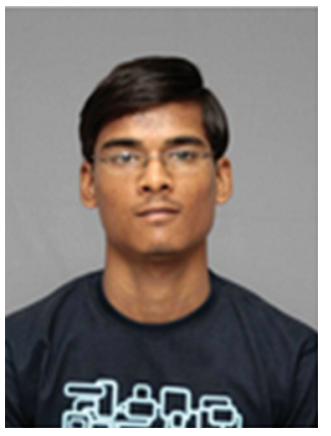

A. Agarwal obtained his B.Tech degree in Electronics and Communications Engineering from VIT University, Vellore, in 2014. Currently he is working as software Engineer. His area of interests include wireless networking.

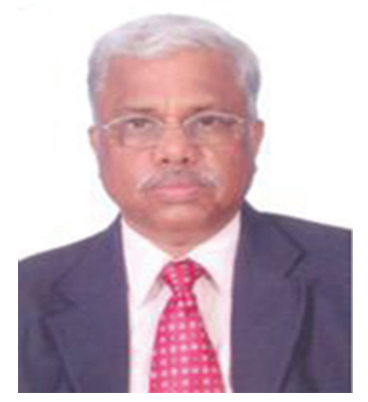

Dr. V. Prithviraj is now the principal of Rajalakshmi Institute of Technology, Chennai. He had served from August 2008-January 2013 as the Principal of Pondicherry Engineering College. He completed his Bachelor of Engineering (Electronics and Communication Engineering) in 1972 from the College of Engineering, Guindy Madras University, his M.S. by research in 1982 from IIT, Madras, and his Ph.D in 1999 from IIT, Kharagpur (research area-Signal Processing Techniques in Array Antennas Systems). He is one of the founding members of Pondicherry Engineering College and served the institute from July 1985-2013. He was holding the position of Dean-in-charge, School of Engineering, Pondicherry University from May 2009-January 2013. 


\section{A Bagubali et al.}

He has been teaching for over 30 years and held the position of Head of the Electronics and Communications Engineering Department at PEC from June 2003-2006. He has published over 80 technical research papers. He has also held the position of Director, IT for Government of Pondicherry (2002-2005). Currently, he is a Member of Expert Committee for monitoring International Indo-French projects in the field of Information Technology as well as Regional Committee of AICTE for Tamil Nadu and Pondicherry. He has a keen interest in research and development projects and provided leadership in many successful projects sponsored by various organizations such as DRDO, ISRO, Department of Electronics and Department of Information Technology at IITs and PEC. He is the recipient of the IEEE International Student Branch Award in 1984 and the EDI Award for best technical paper, entitled "COFDM for Telemedicine Applications", in 2007. He is a Life Member of ISTE and Member of EMC Engineers and IEEE USA. His areas of interest include Broadband and Wireless Communication, Mobile Computing, VLSI for Wireless Applications, Tele Medicine, SDR, Cognitive Radio and e-Governance Applications.

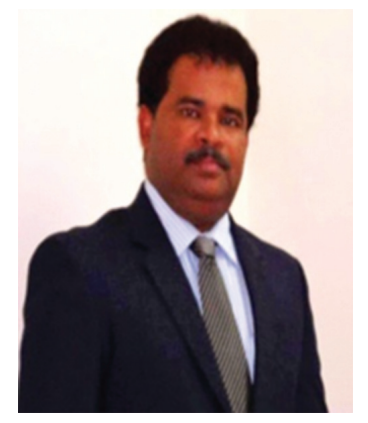

P. S. Mallick, received Ph.D from Jadavpur University, Kolkata, India. He worked 4.5 years in a Sweden based electronics industry named IAAB Electronics as a Technical Head. He has 12 years of Teaching experience where he led various research teams and developed "Online Lab in Microelectronics", "Monte Carlo Simulator for Compound Semiconductors", "Nanostructured MIM Capacitor" and "Low cost Electric Fencer". His current area of research interest includes Nanoscale CMOS, Nanoelectronics and VLSI Engineering. He has published 48 research papers in different Journals and Conferences of International repute and authored a book on Matlab and Simulink. At Present Dr Mallick is working for School of Electrical Engineering, VIT University, Tamilnadu, India, as a Professor and Dean. Dr Mallick has received the 
prestigious Jawaharlal Nehru Scholarship in 1998 for his doctoral research work. He is one of the enlisted technical innovators of India in 2007. He is a Sr. member of IEEE, IEEE-EDS, Life member of IACS, ISTE, and Indian Laser Association. He has successfully completed six research projects funded by the Govt. of India and organized several International Conferences. 
